\title{
The early development of the normative mind
}

\author{
Markus Paulus ${ }^{1} \&$ Marco F. H. Schmidt ${ }^{2}$ \\ ${ }^{1}$ Developmental Psychology, Department of Psychology, LMU Munich, Leopoldstraße 13, \\ 80802 Munich, Germany \\ ${ }^{2}$ International Junior Research Group Developmental Origins of Human Normativity, \\ Department of Psychology, LMU Munich, Leopoldstraße 13, 80802 Munich, Germany
}

\section{Preprint Version}

Final version published in Journal of Experimental Child Psychology

Acknowledgements: Manuscript preparation was supported by grant PA2302/9-1 by the German Research Foundation (Deutsche Forschungsgemeinschaft; DFG) to MP, grant 679000 by the European Research Council (ERC) to MP, and a James S. McDonnell Understanding Human Cognition Scholar Award to MP. MFHS was supported by a grant from the Elite Network of Bavaria, an initiative of the Bavarian State Ministry for Education, Science, and the Arts. 


\begin{abstract}
Normativity is pervasive in everyday human social interactions and perhaps even constitutive of human forms of group and societal living. In the last decade, there has been an increased interest in the ontogeny of normativity and the role norms play in early social reasoning and behavior. Given the ubiquity of normativity, it is vital to investigate the development of children's normative understanding and behavior in a variety of different contexts, ranging from prosocial behavior to rational action, or from linguistic competencies to cultural norms and values. Hence, in this special issue on The early development of the normative mind, researchers from different theoretical traditions have employed a number of different methods (e.g., third-party norm enforcement, judgment and reasoning, social behavior) to address different, yet related, research questions about the ontogeny of normativity. Here, we introduce the reader to the current debate and point to important research questions for the field.
\end{abstract}




\section{The early development of the normative mind}

Norms are a fundamental pillar of human social life (Brandom, 1994; Turiel, 2002), and guide our conduct in virtually any social interaction, be it on an interpersonal, group, or institutional level. Consequently, the development of children's reliance on and understanding of norms has been a longstanding topic in developmental psychology. For example, in his seminal work, Piaget (1932/1965) examined how children construe rules in social games and suggested developmental change in stage-like fashion from egocentric thinking about norms (or rather dictates, i.e., heteronomy) to more cooperative and reflective thinking about norms (autonomy). This line of work was further developed by putting particular emphasis on the ontogeny of moral judgment and individuals' justification for why one should act in certain ways by Kohlberg and colleagues $(1963,1969)$.

Whereas these traditional accounts largely assumed that young children are heteronomously motivated to follow norms (e.g., by relying on adult authorities and fear of punishment) and do not understand much about their context-specific application or even origin, more recent research provided evidence for a hitherto underestimated appreciation of social norms in young children. For instance, a rich body of work by the social domain tradition suggests that preschoolers make systematic distinctions between different types of norms when reasoning about hypothetical situations (e.g., moral, conventional, and personal norms; Killen \& Smetana, 2015; Nucci, 2014). And more recent research suggests that in their social interactions with others, young children proactively enforce norms by criticizing others who violate rules, even if children themselves are not affected by a norm violation (e.g., Rakoczy, Warneken, \& Tomasello, 2008). Further evidence comes from research on children's fairness understanding and behavior. Here, it has been shown that preschool children appreciate fairness norms (Rochat et al., 2009; Smith, Blake, \& Harris, 2013), that

they request and enforce fair behavior by others (Paulus, Gillis, Li, \& Moore, 2013; Rakoczy, 
Kaufmann, \& Lohse, 2016), and that, with age, children increasingly rectify inequality while occasionally also endorsing inequality, if justified (Elenbaas, Rizzo, Cooley, \& Killen, 2016; Paulus, 2014; Schmidt, Svetlova, Johe, \& Tomasello, 2016). Further research on children's understanding of conventional norms, such as game rules, showed that by 3 years of age, children apply rules in context-specific ways and tend to infer them promiscuously based on minimal evidence, such as the intentionality of an agent (e.g., Schmidt, Butler, Heinz, \& Tomasello, 2016). These recent lines of work converge in their demonstration of an early developing motivation for, reliance on, and understanding of social norms.

In addition to work on the normative foundations of prosocial behavior and reasoning about others' welfare (i.e. morality; Paulus, 2014b), scholars pointed to the normative nature of early imitation and social learning (e.g., research on over-imitation; Kenward, 2012), language acquisition, and epistemic activities (Schmidt \& Rakoczy, in press). Across several research areas and domains, there has thus been an increased interest in the normative nature of young children's reasoning, judgment, and behavior. Consequently, it has been suggested that a full understanding of early developmental changes in these domains requires an appreciation of young children's emerging understanding of normativity. In other words, the emerging awareness of normativity has been supposed to play a major role across a number of important domains including imitation, reasoning and judgment in social contexts, language learning, and (pro)social behavior.

\section{Important questions about the developing normative mind}

Thus, recent years have seen considerable advance in theory and research on children's developing normative mind and behavior. At the same time, there are more and more exciting new questions arising from both classical and new findings as well as from attempts to integrate research on normativity with other domains and topics of investigation. 
For example, important questions pertain to how normative reasoning and behavior develops in contexts involving multiple issues (e.g., on an individual and group level) and domains (e.g., moral and conventional norms), given that such situations mimic children's real-life experience to a large degree. For instance, how do children balance individual, group, and moral concerns?

Moreover, the norms of the social world differ in important aspects from the laws of the physical world (Brandom, 1994). Yet, little is known on how young children come to differentiate between those (e.g., Kalish, 1998). That is, although much research suggests that children begin early to categorize, apply, and reason about norms, it is not clear how far their differentiation of norms or even meta-understanding of rules as social constructs goes, how their understanding develops, and what (social-)cognitive abilities allow for, or contribute to, children's developing normative mind.

What is more, norms, values, language, and culture are intimately linked constructs that most likely refer to reciprocal processes in the development of children's understanding of normativity - so far, however, there has been little research on the interplay of these processes.

This special issue of the Journal of Experimental Child Psychology aims at addressing several of these exciting new avenues and questions on the early ontogeny of the normative mind. In what follows, we briefly introduce the contributions of this special issue.

\section{Contributions in the current special issue}

This special issue of the Journal of Experimental Child Psychology contains several contributions that, coming from different theoretical traditions, address research questions that all pertain to the early developing normative mind. Given the variety of different theoretical perspectives and questions, the contributions employ different methods and tackle a number of important domains that are all relevant to our understanding of the developing normative 
mind. The empirical findings and diverse theoretical perspectives of this special issue may help spur further research on the developmental foundations of the ontogeny of normativity.

First, four articles assess children's judgment, expectations, decisions, and social behavior in intergroup, moral, and conventional contexts.

Liberman, Howard, Vasquez, and Woodward (2017) investigated 3- to 11-year-old children's expectations as to who is more likely to break conventional and moral norms: an ingroup member or an outgroup member? They found that children at all ages expected that ingroup members will conform to conventional norms whereas outgroup members may be more likely to violate these types of norms. Only by around 7 years of age, however, did children make a difference for moral norms: they expected ingroup members to be less likely than outgroup individuals to break moral norms.

Rizzo, Cooley, Elenbaas, and Killen (2017) investigated 3- to 6-year-olds' decisions about which peer (a dissident ingroup member who challenges an ingroup norm vs. an outgroup member who supports the ingroup norm) to include in moral and conventional contexts. They found that in moral contexts (involving issues about distributive justice), children focused on the norm of equality and neglected group membership when including a peer into their group. In conventional contexts, however, children were more likely to focus on issues of conformity (in particular, conformity with group-level traditions).

Wilks and Nielsen (2017) assessed the stability of preschoolers' and early school-aged children's ingroup favoritism in contexts in which ingroup members behaved antisocially and were paired with outgroup members who behaved prosocially or antisocially. Older, but not younger, children took antisocial ingroup behavior into account, such that their ingroup preference diminished and they tended to like their own group less when they witnessed ingroup members behaving antisocially. Nonetheless, children themselves did not take antisocial ingroup behavior as a reason to allocate resources unequally between the ingroup and the outgroup. 
Harvey, Davoodi, and Blake (2017) investigated 5- to 8-year-old children's tendency to lie to prevent moral transgressions from happening. Children were given the opportunity to misdirect an agent who, for instance, intended to steal someone else's toy. The results showed that children were most likely to lie in contexts in which both harm to the victim and the transgression (e.g., stealing) were made salient.

Second, three papers provide novel evidence for children's early developing understanding of norms that pertain to issues of charity, instrumental rationality, and norm change.

Wörle and Paulus (2017) assessed the emergence of a norm of charity (i.e. the obligation to give more to poor others than to rich others) in preschool children. They found that 5- to 6-year-old children, but not 3- to 4-year-old children selectively protested against an agent who distributed more resources to a rich recipient, and affirmed an agent who distributed more resources to a poor recipient. The younger children, in contrast, rather seemed to adhere to a norm of equal distribution.

Dahl and Schmidt (2017) investigated preschoolers' (and adults') understanding of norms of instrumental rationality (i.e., the norm to use effective means to reach one's ends) and assessed whether participants treat them as dependent on individual goals (hypothetical imperatives) or as valid independent of individual goals (categorical imperatives). They found that children, but not adults, understood instrumental norms (as well as moral and prudential norms) as categorical imperatives that apply independent of what an agent wants.

Nonetheless, children made systematic distinctions between instrumental and other types of norms.

Zhao and Kushnir (2017) presented 4- to 7-year-old participants with children playing game together and manipulated whether the game rules were set by an adult, the entire group, or a single child. When the rules were created by one person (adult or child), participants thought that only this individual could change the rules. Yet, when the entire group agreed on 
a rule, younger children believed that any member of the group could change them, whereas older children thought that no group member could do that. Thus, young children seem to consider the context of norm creation when considering who is able to change a norm.

Third, three articles pertain to questions of how culture and values are related to the developing normative mind. The contributions investigated interrelations between parental or child values and children's own (prosocial or norm enforcement) behavior, as well as children's tendency to promiscuously infer norms from descriptive facts.

Abramson, Daniel, and Knafo-Noam (2017) investigated the relation between 5- to 12-year-old children's values and their sharing behavior. They showed that children have a hierarchical structured representation of values and that their appreciation of selftranscendence related values is in line with their actual sharing behavior.

Gampe and Daum (2017) investigated interrelations between parental cultural values and bicultural children's reactions to game rule violations. They found that 3-year-old children's reactions to rule violations (e.g., protest or more indirect forms of communication, such as questions) were related to parental values (in particular, institutional collectivism, assertiveness, and performance orientation), suggesting potential links between parental cultural attitudes and children's behavior in norm-relevant contexts.

Roberts, Guo, Ho, and Gelman (2017) asked whether children's (and adults') tendency to make normative and evaluative judgments about behaviors that violate (descriptive) group regularities can be found in Chinese children. They found that children disapproved of nonconforming behaviors, much like U.S. children, but that younger Chinese children's disapproval was particularly pronounced in comparison with U.S. children. Furthermore, Chinese adults - in contrast to U.S. adults - tended to evaluate non-conformity to group regularities more negatively than conformity.

And fourth, two papers investigate relations between language and normativity, looking at children's modal cognition and their understanding of the generic "you". 
Shtulman and Phillips (2017) examined to which extent 3- to 10-year-old children and adults differentiate between impossible, improbable, unconventional, and immoral events. The results showed that preschool children have difficulties in distinguishing events that violate norms compared to events that go against regularities. In addition, preschoolers evaluated physically unlikely events as immoral and immoral behavior as impossible, demonstrating that preschoolers have problems distinguishing between the social world of norms and the world of physical laws.

Orvell, Gelman, and Kross (2017) assessed 2- to 10-year-old children's understanding and use of the generic „you“ as an indicator of their normative understanding. They showed that by the preschool years, children distinguish between the canonical and generic use of „you“ indicating a differentiation between norms and preferences.

Together, the contributions in this special issue shed light on the early development of norms and some of the factors and abilities that help children to become competent members of their culture. The findings and theoretical perspectives of this issue will be of great benefit for future research and attempts to unravel the ontogeny of the normative mind. 


\section{References}

Abramson, L., Daniel, E., \& Knafo-Noam, A. (2017). The role of personal values in children's costly sharing and non-costly giving. Journal of Experimental Child Psychology. Doi: https://doi.org/10.1016/j.jecp.2017.03.007

Brandom, R. B. (1994). Making it explicit. Cambridge, Mass.: Harvard University Press.

Dahl, A., \& Schmidt, M. F. H. (in press). Preschoolers, but not adults, treat instrumental norms as categorical imperatives. Journal of Experimental Child Psychology.

Elenbaas, L., Rizzo, M. T., Cooley, S., \& Killen, M. (2016). Rectifying social inequalities in a resource allocation task. Cognition, 155, 176-187. Doi: https://doi.org/10.1016/j.cognition.2016.07.002

Gampe A., \& Daum, M. M. (2017). How preschoolers react to norm violations is associated with culture. Journal of Experimental Child Psychology. Doi: https://doi.org/10.1016/j.jecp.2017.06.009

Harvey, T., Davoodi, T., \& Blake, P. R. (2017). Young children will lie to prevent a moral transgression. Journal of Experimental Child Psychology. Doi: https://doi.org/10.1016/j.jecp.2017.06.004

Kalish, C. (1998). Reasons and causes: Children's understanding of conformity to social rules and physical laws. Child Development, 69, 706-720. Doi: https://doi.org/10.1111/j.1467-8624.1998.tb06238.x

Kenward, B. (2012). Over-imitating preschoolers believe unnecessary actions are normative and enforce their performance by a third party. Journal of Experimental Child Psychology, 112(2), 195-207. Doi: https://doi.org/10.1016/j.jecp.2012.02.006

Killen, M., \& Smetana, J. G. (2015). Origins and development of morality. In R. M. Lerner (Ed.), Handbook of child psychology and developmental science (7th ed., Vol. 3, pp. 701-749). NY: Wiley-Blackwell. 
Kohlberg, L. (1963). The Development of Children's Orientations Toward a Moral Order. Human Development, 6(1-2), 11-33.

Kohlberg, L. (1969). Stage and sequence: The cognitive-developmental approach to socialization. In D. A. Goslin (Ed.), Handbook of socialization theory and research (pp. 347-480). Chicago, IL: Rand McNally.

Liberman, Z. Howard, L. H., Vasquez, N. M., \& Woodward A. L. (2017). Children's expectations about conventional and moral behaviors of ingroup and outgroup members. Journal of Experimental Child Psychology. Doi: https://doi.org/10.1016/j.jecp.2017.03.003

Nucci, L. P. (2014). The personal and the moral. In M. Killen \& J. G. Smetana (Eds.), Handbook of moral development (Second edition, pp. 538-558). New York, NY: Psychology Press.

Orvell, A., Kross, E., \& Gelman, S. A. (2017). That's how “you” do it: Generic you expresses norms during early childhood. Journal of Experimental Child Psychology. Doi: https://doi.org/10.1016/j.jecp.2017.04.015

Paulus, M. (2014a). The early origins of human charity: developmental changes in preschoolers' sharing with poor and wealthy individuals. Frontiers in Psychology, 5, 344. Doi: https://doi.org/10.3389/fpsyg.2014.00344

Paulus, M. (2014b). The emergence of prosocial behavior: Why do infants and toddlers help, comfort, and share? Child Development Perspectives, 8, 77-81. Doi: https://doi.org/10.1111/cdep. 12066

Paulus, M., Gillis, S., Li, J., \& Moore, C. (2013). Preschool children involve a third party in a dyadic sharing situation based on fairness. Journal of Experimental Child Psychology, 116(1), 78-85. Doi: https://doi.org/10.1016/j.jecp.2012.12.014

Piaget, J. (1965). The moral judgment of the child. (M. Gabain, Trans.). New York, NY: Free Press. (Original work published 1932). 
Rakoczy, H., Brosche, N., Warneken, F., \& Tomasello, M. (2009). Young children's understanding of the context relativity of normative rules in conventional games. British Journal of Developmental Psychology, 27, 445-456. Doi: https://doi.org/10.1348/026151008X337752

Rakoczy, H., Kaufmann, M., \& Lohse, K. (2016). Young children understand the normative force of standards of equal resource distribution. Journal of Experimental Child Psychology, 150, 396-403. Doi: https://doi.org/10.1016/j.jecp.2016.05.015

Rakoczy, H., Warneken, F., \& Tomasello, M. (2008). The sources of normativity: young children's awareness of the normative structure of games. Developmental Psychology, 44(3), 875-81. Doi: https://doi.org/10.1037/0012-1649.44.3.875

Rizzo, M. T., Cooley, S., Elenbaas, L., \& Killen, M. (2017). Young children’s inclusion decisions in moral and social-conventional group norm contexts. Journal of Experimental Child Psychology. Doi: https://doi.org/10.1016/j.jecp.2017.05.006

Roberts S. O., Guo, C., Ho, A. K., \& Gelman S. A. (2017). Children’s descriptive-toprescriptive tendency replicates (and varies) cross-culturally: Evidence from China. Journal of Experimental Child Psychology. Doi: https://doi.org/10.1016/j.jecp.2017.03.018

Rochat, P., Dias, M. D. G., Liping, G., Broesch, T., Passos-Ferreira, C., Winning, A., \& Berg, B. (2009). Fairness in Distributive Justice by 3- and 5-Year-Olds Across Seven Cultures. Journal of Cross-Cultural Psychology, 40(3), 416-442.

Schmidt, M. F. H., Butler, L. P., Heinz, J., \& Tomasello, M. (2016). Young children see a single action and infer a social norm: Promiscuous normativity in 3-year-olds. Psychological Science, 27(10), 1360-1370. Doi: https://doi.org/10.1177/0956797616661182

Schmidt, M. F. H., \& Rakoczy, H. (in press). Developing an understanding of normativity. In A. Newen, L. de Bruin, \& S. Gallagher (Eds.), Oxford Handbook of Cognition: 
Embodied, Embedded, Enactive and Extended. Oxford: Oxford University Press (to appear in 2018).

Schmidt, M. F. H., Rakoczy, H., \& Tomasello, M. (2011). Young children attribute normativity to novel actions without pedagogy or normative language. Developmental Science, 14(3), 530-539. Doi: https://doi.org/10.1111/j.1467-7687.2010.01000.x

Schmidt, M. F. H., Svetlova, M., Johe, J., \& Tomasello, M. (2016). Children’s developing understanding of legitimate reasons for allocating resources unequally. Cognitive Development, 37, 42-52. Doi: https://doi.org/10.1016/j.cogdev.2015.11.001

Smith, C. E., Blake, P. R., \& Harris, P. L. (2013). I should but I won’t: Why young children endorse norms of fair sharing but do not follow them. PLoS ONE, 8(3), e59510. Doi: https://doi.org/10.1371/journal.pone.0059510

Shtulman, A., \& Phillips, J. (2017). Differentiating “could" from "should": Developmental changes in modal cognition. Journal of Experimental Child Psychology. Doi: https://doi.org/10.1016/j.jecp.2017.05.012

Turiel, E. (2002). The culture of morality: Social development, context, and conflict. Cambridge, England: Cambridge University Press.

Wilks, M., \& Nielsen, M. (2017). Children disassociate from antisocial in-group members. Journal of Experimental Child Psychology. Doi: https://doi.org/10.1016/j.jecp.2017.06.003

Wörle, M., \& Paulus, M. (2017). Normative expectations about fairness: The development of a charity norm in preschoolers. Journal of Experimental Child Psychology. Doi: https://doi.org/10.1016/j.jecp.2017.03.016

Zhao, X., \& Kushnir, T. (2017). Young children consider individual authority and collective agreement when deciding who can change rules. Journal of Experimental Child Psychology. Doi: https://doi.org/10.1016/j.jecp.2017.04.004 